\title{
Energy Flow Hybrid Petri Nets Model of Steel Enterprises
}

\author{
ZHAO Ye-Qing \\ School of Computer and Information Engineering, Anyang Normal University, Anyang,China
}

\begin{abstract}
As the urgency of current energy-saving and discharge reduction task and difficult of related advance technology in breaking through, a new energy flow network system model was built based on modelling elements and rules of hybrid Petri nets to adapt to the system requirements through analyzing the basis features of energy flow network, and meet the demand of developing energy flow network efficiency of iron and steel enterprises. The dynamic rules and behaviour mechanism of energy flow network system for iron and steel enterprises were studied by utilizing the model combined with the historical data, the results show that the modelling method is effective and satisfactory, and provide guidance for optimizing the energy system and exploring the relation of material flow and energy flow.
\end{abstract}

\section{Introduction}

Scholars and entrepreneurs at home and abroad have done a great deal of work on energy saving of iron and steel enterprises. Through the continuous development of theoretical and practical research work, academician Lu takes the system of iron and steel as the research object, and set up the theory of energy saving of industrial system by putting forward to a series of concepts and methods (Lu, 1996;2000). On account of complex characteristics of the material flow and energy flow in production process of the iron and steel, Lu put forward the concept models of standard materials flows diagram, standard energy consumption per ton and standard metal yield per ton, and establish the basic method for analyzing the impact the change of material flow on the energy consumption of each stock in the steel production process (Lu, 2000). Professor Cai built the energy saving decision making model of iron and steel enterprises by using the large system optimization method, and realized the optimization of the production system of Anshan Iron and Steel Group and get a more reasonable production plan (Cai,2012). The nature of production process is the flow process of material flow driven by energy flow in iron and steel enterprises, Academician Yin studied the essence and law of iron and steel production process from microcosmic to macroscopic aspects, and provided a method and breakthrough point for energy saving and consumption reduction of iron and steel system(Yin,1997;2000). Many foreign scholars have analyzed the energy consumption problem of industry or enterprise by applying the exergy analysis method of the second law of thermodynamics (Larsson, 2006; Polenske, 2002; Andersen, 2014). In the light of the specific case, the practical implementation scheme was put forward to achieve a certain optimization effect. The above modeling method either cannot optimize the whole energy system by taking a specific equipment and specific process of steel enterprise as the research object, or cannot effectively reflect the characteristics of the dynamic and orderly and compact system of the actual energy system in iron and steel enterprises by taking the production system as the object and maximizing the energy saving of the system as a goal. Further, the above modeling method cannot be used to establish the energy flow network model for lack of the information interaction of different media energy flow networks and directly energy dynamic regulation.

In this paper, based on the idea of network of energy flow, we discuss the building methodology of energy flow network by analyzing the distribution and input/output characteristics of the complex energy flow system in iron and steel enterprises, and build the energy flow network model of three links including energy use, energy recovery and energy conversion in the whole process based on hybrid Petri nets. Then combined with the historical data, the dynamic energy network system model for iron and steel enterprises is carried out to verify the correctness and effectiveness of the energy flow hybrid Petri nets model(EFHPNM), and lay a solid foundation for achieving the dynamic, efficient and orderly operation of the enterprise energy system.

\section{Energy Flow Characteristics Of System Network In Steel Enterprises}

Modern steel enterprise is a typical complex process system of chemical process and deep processing of iron coal. On the one hand, iron and other raw materials need

\footnotetext{
*Corresponding author: zhaoyq0603@163.com
} 
to undergo a series of physical and chemical changes to achieve the transformation from raw materials to products, and this process is expressed as the form of material flow. On the other hand, carbon and other energy materials can transform energy products through a series of processes such as processing, transformation and On the other hand, carbon materials and other energy materials can transform energy products through a series of processes such as processing, transformation and modification, and this process is expressed as the form of energy flow. In the steel manufacturing process, ferrite materials flow is accompanied by carbon energy flow, and carbon energy flow drive ferrite materials flow in particular process network to do orderly dynamic movement for achieving multi-objective optimization of the whole system. But in the whole system dynamic operation, because of the energy flow and material flow sometimes coupling and sometimes separating, the materials flow and the energy flow have their own process networks.

In order to make the energy flow network model of the steel enterprise more effectively and more suitable for the actual enterprises, we must combine the advantages of modelling tools with the characteristics of modelling objects. Based on the inherent characteristics of materials flow and energy flow networks system in iron and steel enterprises, this paper uses hybrid Petri nets to realize the modelling of energy flow networks system based on the modelling methods and theory of hybrid Petri nets.

\section{Hybrid Petri Net Model Of Energy Flow Network System}

Petri net is a graphical modeling tool, which not only has strict mathematical definition, but also provides system methods of analysis and verification. It is the main method of modeling for analyzing dynamic system of discrete event[14-17].Hybrid Petri nets is Petri net model that can realize discrete event system and continuous event system in direct interaction, visual representation, qualitative and quantitative analysis, real-time control and simulation analysis at the same level[18-20].In the hybrid Petri nets model, Petri net is composed of discrete and continuous parts, two parts are connected by connecting arcs (edges) between a discrete node and a continuous node, and that the discrete parts and the continuous parts in the dynamic system can affect and interact each other.

\subsection{Definition Of Hybrid Petri Nets}

Definition 1 Continuous Petri net is a six-tuple $C O P N=\left(P, T, F, W, V, M_{0}\right)$, where:

(1) $P$ is the nonempty sets, which means the continuous place and expressed as (O);

(2) $T$ is the nonempty sets, which means the continuous transition and expressed as \;
(3) $F \subseteq P \times T \cup T \times P$ is a set of directed arcs, which connected from the continuous transition to the continuous place, and vice versa;

(4) $W=\{$ Pre, Post $\}$ is the weight of the directed arc;

(5) $V$ is the velocity of continuous transition;

(6) $M$ is the marking matrix and $M_{0}$ is the initialization marking matrix.

Definition 2 hybrid Petri nets is six-tuple HPNs $=(P, T, \operatorname{Pr} e$, Post $, h, M)$,where:

(1) $P=P^{D} \cup P^{C}=\left\{p_{i}, i=1,2, \cdots, m\right\}, P^{C}$ is the sets of continuous place and $P^{D}$ is the sets of discrete place;

(2) $T=T^{D} \cup T^{C}=\left\{t_{i}, i=1,2, \cdots, n\right\}, T^{C}$ is the sets of continuous transition and $T^{D}$ is the sets of discrete transition;

(3) Pre is the weight of the arc between the transition $t_{j}$ and its input place $p_{i}$, which defined as follows:

$$
\operatorname{Pr} e_{i, j}:(P \times T) \rightarrow\left\{\begin{array}{l}
R^{+}, \forall p_{i} \in P^{C} \\
N^{+}, \forall p_{i} \in P^{D}
\end{array}\right.
$$

(4) Post is the weight of the arc between the transition $t_{j}$ and its input place $p_{i}$, which defined as follows:

$$
\text { Post }_{i, j}:(P \times T) \rightarrow\left\{\begin{array}{l}
R^{+}, \forall p_{i} \in P^{C} \\
N^{+}, \forall p_{i} \in P^{D}
\end{array}\right.
$$

(5) $h: P \cup T \rightarrow\{D, C\}$ is the mixed function, which indicates that the type of node is discrete(D) or continuous $(\mathrm{C})$;

(6) $M$ is the marking function, which defined as follows:

$$
M: P \rightarrow\left\{\begin{array}{l}
R^{+}, \forall p_{i} \in P^{C} \\
N^{+}, \forall p_{i} \in P^{D}
\end{array} .\right.
$$

\subsection{Hpns Model Of Energy Flow Networks System}

In order to model the energy flow network system by using HPNs, the rules of mapping the energy flow network system to the HPN model are given as follows:

(1)The storage and transportation equipment of energy networks system in the steel enterprise is represented by a continuous palace, such as BFG holder, CFG holder and so on, which is expressed as (0) .

(2)The process of energy output and consumption in the processes and stations is represented by a continuous translation, which is expressed as $\mathbf{\text { . }}$

(3)The working state (start and stop) of the stations in the processes is represented by a discrete palace, which is expressed as $\bigcirc$. 
(4)The operation of the launching and terminating for the station in the processes and the corresponding approval are represented by discrete translation, which is expressed as $\square$.

(5)The connection between palace and translation indicates the output or consumption of energy system, which is represented by directed arc.

Owning to the complexity of the energy flow networks system in the steel production process, the idea of hierarchical modelling is adopted to realize the effective modelling of complex system by using HPNs, and reducing the complexity of the model. The continuous translation can be composed of a series of sub-networks, and the sub-network can be further nested. That is to say, the continuous translation can be decomposed into several sub-networks made of stations and production equipment units. Firstly, the top layer (home page) HPNs model of the energy flow networks system is established, as shown in figure 1 (p01,p02,..,p12 are the discrete place, which mean the enabled instruction of energy subsystem and the status;P99 is the energy system start up instructions; p21,p22,..,p26 are continuous place, which mean gas network, power grid, steam network, gas holder, compressed gas tank and gas-pipe network;t11,t12,..,t16 are discrete translation, which mean start action of energy subsystem;t $21, \mathrm{t} 22, \ldots, \mathrm{t} 26$ are continuous translation, which mean the subsystem such as fuels, power, steam, technical gas, compressed gas and coal gas).Then, the(sub page) HPNs model of each complex continuous translation is constructed respectively. Figure 2 shows the HPN model of the gas energy flow network system in iron and steel enterprises.

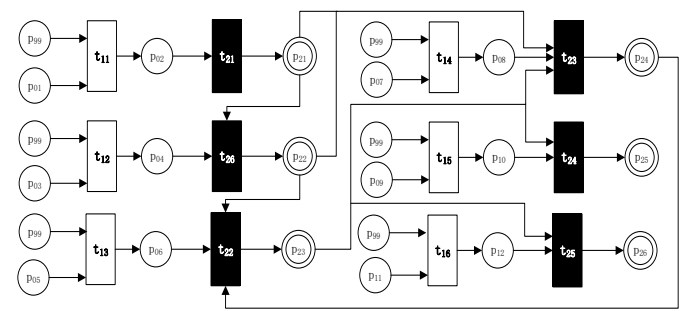

Figure 1: Energy flow network system main HPN model.

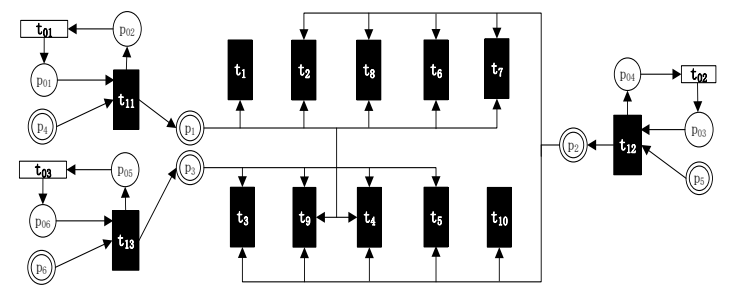

Figure 2: Gas energy flow network system subordinate HPN model.

\section{Modeling And Analysis}

In order to make the system model with elegant simplicity, Extendsim adapts hierarchical and modular structure to realize the modeling of large complex systems .In addition, its rich graphic output function is sufficient to meet the user's output requirements.
According to the actual production process of the energy system in a steel plant, we built the energy flow network system HPNs model based on the of simulation platform Extendsim.

The blast furnace gas(BFG), coke oven gas (COG) and converter gas (LDG) is the byproduct gas of iron and steel enterprises, which are the most important secondary energy and accounted for about $40 \%$ of the energy in the steel enterprises. The gas networks system is associated with almost all the processes and key positions, so it is the most important sub-networks system of the energy networks system in the iron and steel enterprises. Next, the gas energy flow networks system is simulated and other energy flow networks system are omitted because of the length of the article.

Based on analyzing historical productive data and combining with current reality environment, the HPNs model of energy flow networks system in the steel enterprises was simulated by using the optimization module of simulation platform Extendsim, the comparison results for the production data and simulation data of gas networks system is showed in Figure 3, the PDA stands for the actual production data of the gas networks system in the production process of steel enterprises, and the SDA stands for the simulation data of the gas networks system in the production process of steel enterprises. As can be seen from Figure 3, compared with the historical value, the energy flow density of gas energy flow network system in the production process of steel enterprises has a better effect (maximum increment $10 \%$, minimum increment $2.5 \%$, average increment $5.45 \%$ ) by optimizing the system, so the gas energy flow network system in the production process of steel enterprises can be further adjusted.

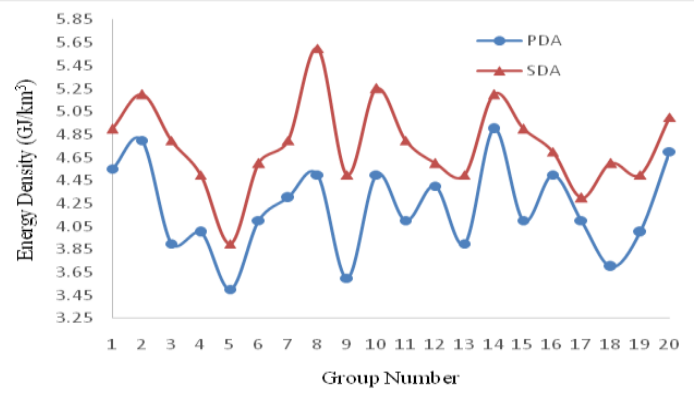

Figure 3: Gas energy flow density contrast figure.

\section{Conclusions}

The energy flow networks system model for the iron and steel enterprises is built based on stratification theory and formal modeling tools Petri nets. Through the simulation of the energy flow networks system, we prove that the validity and correctness of the modelling methods based on hybrid Petri nets and gas sub-networks system analysis. Thus, the energy flow networks system HPNs model can better describe the system characteristics of mobility, dynamics, and complexity. Based on the actual situation and the statistical 
analysis of historical data for steel-making, the correctness and validity of the modeling method is verified by simulation, and the results provide theoretical and practical guidance for us to adjust and optimize the energy networks system in iron and steel enterprises.

\section{Acknowledgements}

This work is supported by the key scientific and technological project of Henan province under Grant nos.172102210168 and 16A520001.

\section{References}

1. Lu, Z., Xie, A., \& Zhou, D., 1996. More on the directions and measures of energy conservation of chinese steel industry. Iron \& Steel.

2. Lu, Z., Cai, J., Qingbo, Y. U., \& Xie, A. ,2000. The influences of materials flows in steel manufacturing process on its energy intensity. Acta Metallrugica Sinica.

3. Cai, J. J., Sun, W. Q.,2012. Systems energy conservation and scientific energy utilization of iron and steel industry in china. Iron \& Steel, 47(5), 1-8.

4. Yin, R.,1997. The multi-dimensional mass-flow control system of steel plant process. Acta Metallrugica Sinica, 33(1), 29-38.

5. Yin, R.,2000. Analysis and integration of steel manufacturing process. Acta Metallrugica Sinica.

6. Larsson, M., Wang, C., \& Dahl, J.,2006. Development of a method for analysing energy, environmental and economic efficiency for an integrated steel plant. Applied Thermal Engineering, 26(13), 1353-1361.

7. Polenske, K. R., \& Mcmichael, F. C.,2002. A chinese cokemaking process-flow model for energy and environmental analyses. Energy Policy, 30(10), 865-883.

8. Andersen, J. P., \& Hyman, B.,2014. Energy and material flow models for the us steel industry. Energy, 26(2), 137-159. 